\title{
Damping Acoustic Modes in Compressible Horizontally Explicit Vertically Implicit (HEVI) and Split-Explicit Time Integration Schemes
}

\author{
Joseph B. Klemp, William C. Skamarock, And Soyoung Ha \\ National Center for Atmospheric Research, Boulder, Colorado
}

(Manuscript received 15 December 2017, in final form 9 April 2018)

\begin{abstract}
Although the equations of motion for a compressible atmosphere accommodate acoustic waves, these modes typically play an insignificant role in atmospheric processes of physical interest. In numerically integrating the compressible equations, it is often beneficial to filter these acoustic modes to control acoustic noise and prevent its artificial growth. Here, a new technique is proposed for filtering the $3 \mathrm{D}$ divergence that may damp acoustic modes more effectively than filters previously implemented in numerical modes using horizontally explicit vertically implicit (HEVI) and split-explicit time integration schemes. With this approach, a divergence damping term is added as a final adjustment to the horizontal velocity at the new time level after completing the vertically implicit portion of the time step. In this manner, the divergence used in the filter term has exactly the same numerical form as that used in the discrete pressure equation. Analysis of the dispersion equation for this form of the filter documents its stability characteristics and confirms that it effectively damps acoustic modes with little artificial influence on the amplitude or propagation of the gravity wave modes that are of physical interest. Some specific aspects of the implementation of the filter in the Model for Prediction Across Scales (MPAS) are discussed, and results are presented to illustrate some of the beneficial aspects of suppressing acoustic noise.
\end{abstract}

\section{Introduction}

While acoustic modes are present in the compressible atmosphere, they typically play an insignificant role in atmospheric processes of physical interest. This recognition has led to several different approaches in designing the numerics for nonhydrostatic simulation models. One technique is to remove acoustic modes from the governing equations by solving a modified anelastic set of equations, which requires the solution of a 3D Poisson equation using an implicit solver at each time step (e.g., Ogura and Phillips 1962; Wilhelmson and Ogura 1972; Lipps and Hemler 1982; Kurowski et al. 2014). A second approach is to solve the compressible equations using a semi-implicit scheme, resulting in a 3D Helmholtz equation for pressure that also must be solved using implicit numerics at each time step (e.g., Tapp and White 1976; Tanguay et al. 1990; Staniforth and Côté 1991). This approach stabilizes the acoustic modes by essentially retarding their frequencies. A third alternative numerical procedure treats the compressible equations by solving the terms responsible

Corresponding author: Dr. Joseph B. Klemp, klemp@ucar.edu for vertical acoustic propagation using semi-implicit numerics, while solving for the terms accommodating horizontal acoustic propagation using split-explicit or horizontally explicit vertically implicit (HEVI) schemes (e.g., Klemp and Wilhelmson 1978; Klemp et al. 2007; Lock et al. 2014). This approach avoids the need for computationally expensive 3D implicit solvers and does not intermingle higher-frequency acoustic modes with lower-frequency modes of physical interest. However, care must be taken with this approach to ensure that energy does not artificially accumulate in these acoustic modes due to initialization or physics imbalances, nonlinear interactions, or model numerics. In this paper, we focus on techniques to filter acoustic modes through 3D divergence damping for this latter category of schemes, in which horizontally propagating acoustic modes are treated with explicit numerics. HEVI/ split-explicit schemes are widely used in research and operational atmospheric models, and most of these models employ 3D divergence damping to control acoustic noise [e.g., MM5 (Dudhia 1993), COAMPS (Hodur 1997), ARPS (Xue et al. 2000), WRF (Klemp et al. 2007), Nonhydrostatic Icosahedral Atmospheric Model (NICAM; Satoh et al. 2008), LM/COSMO 
(Doms and Baldauf 2015), Model for Prediction across Scales (MPAS; Skamarock et al. 2012), and Icosahedral Nonhydrostatic GCM (ICON; Zängl et al. 2015)]. We are particularly interested in the design of acoustic filtering for the split-explicit numerics in MPAS, which employs a variable-resolution global mesh based on centroidal Voronoi tessellations.

In an early implementation of split-explicit numerics for integrating the compressible equations of motion, Klemp and Wilhelmson (1978) solved the vertically implicit terms using a centered Crank-Nicolson scheme that does not create numerical diffusion. Recognizing the need to filter acoustic modes, Durran and Klemp (1983) introduced a small forward centering of the vertically implicit numerics and verified that the damping achieved on the small acoustic time steps had little effect on the gravity wave modes of interest. This means of acoustic filtering is not entirely effective, however, as it does not affect acoustic modes that have little vertical structure. To more effectively damp acoustic modes in split-explicit solvers, Skamarock and Klemp (1992, hereafter SK92) proposed an explicit filter on the full 3D divergence to augment the filtering of vertical acoustic modes provided by off-centering the vertically implicit numerics. Analyzing the linear compressible Boussinesq equations [see Durran (2010), p. 409], they demonstrated that the explicit damping of 3D divergence can provide effective attenuation of acoustic modes with negligible effect on the gravity wave modes. Acoustic filters based on the SK92 approach have been implemented in the horizontal momentum equations in the WRF Model (Skamarock et al. 2008) and MPAS (Skamarock et al. 2012) and have proven effective in controlling acoustic noise.

Gassmann and Herzog (2007, hereafter GH07) evaluated the compressible linear 2D acoustic/gravity wave equations in analyzing the divergence-damping characteristics for the numerics implemented in the splitexplicit German Weather Service Lokal-Modell (LM) model (see also Gassmann 2005). Their analysis suggested that divergence damping for the compressible equations should be applied in both the horizontal and vertical momentum equations to avoid undesirable effects on the gravity wave modes (i.e., phase errors in the gravity wave frequencies). Therefore, we begin in section 2 by documenting the suitability of employing divergence damping for the fully compressible system only in the horizontal momentum equations, provided the divergence to be filtered is suitably defined. In recent work with MPAS, some issues have arisen with regard to the divergence damping, particularly in applications on variable-resolution meshes, that have prompted a redesign of our techniques for horizontal acoustic-mode filtering. In section 3, we propose a new approach for horizontal damping of the full divergence in a numerically consistent manner and document its impact on both the acoustic and gravity wave modes in conjunction with the off-centering employed in the vertically implicit portion of the solver. For simplicity, the analysis of these numerical filters will be conducted for HEVI schemes to avoid the added complication of the split-explicit numerics. In section 4, we discuss how this acoustic filter is implemented in MPAS and present results from MPAS simulations in section 5 to illustrate its behavior.

\section{Filtering horizontally propagating acoustic modes}

In analyzing divergence damping terms included in the fully compressible linear equations, GH07 obtained a dispersion equation that differed from that obtained by SK92 and suggested that the divergence damping must be applied in both the horizontal and vertical to avoid adversely impacting the gravity wave modes. They concluded that the discrepancy from SK92 arose because SK92 analyzed the compressible Boussinesq equation instead of the fully compressible equations. In their equations, GH07 applied the divergence damping terms using a divergence defined as $D=\nabla \cdot \mathbf{v}$. However, the adiabatic compressible pressure equation (ignoring horizontal advection), written in the form

$$
\frac{\partial p}{\partial t}+\frac{c^{2}}{\theta} \nabla \cdot \rho \theta \mathbf{v}=0
$$

suggests that $D=\nabla \cdot \rho \theta \mathbf{v}$ is a more appropriate expression for the divergence that should be damped in filtering acoustic modes. [Here, $c^{2}=\left(c_{p} / c_{v}\right) R T$ is the square of the sound speed.] This is also consistent with Durran's (1989) finding that the adiabatic pseudoincompressible equation $\nabla \cdot \bar{\rho} \bar{\theta} \mathbf{v}=0$ provides a more accurate representation of gravity waves than other conventional forms of the anelastic continuity equation. Here, the overbars refer to vertically varying mean state profiles.

To confirm the benefit of damping $\nabla \cdot \rho \theta \mathbf{v}$ to suppress the acoustic modes, we rederive the linear dispersion equation for a compressible atmosphere that includes damping using this representation of the divergence. Here, we cast the linear equations in terms of flux-form variables, as described in the appendix of Klemp et al. (2007), which are comparable to the flux-form prognostic variables used in the WRF Model and in MPAS. We emphasize, however, that the resulting linear dispersion equation is independent of the choice of prognostic variables. In terms of the flux-form variables 
$\rho, U=\rho u, W=\rho w$, and $\Theta=\rho \theta$, the 2D linear adiabatic equations (without advection or rotation), including divergence damping terms as proposed by SK92 with the more general expression for divergence, are given by

$$
\begin{aligned}
U_{t}^{\prime}+\frac{c^{2}}{\bar{\theta}} \Theta_{x}^{\prime} & =\frac{\gamma_{h}}{\bar{\theta}} D_{x}^{\prime}, \\
W_{t}^{\prime}+\frac{c^{2}}{\bar{\theta}}\left(\Theta_{z}^{\prime}-\frac{N^{2}}{g} \Theta^{\prime}\right)+g \rho^{\prime} & =\frac{\gamma_{v}}{\bar{\theta}} D_{z}^{\prime}, \\
\rho_{t}^{\prime}+U_{x}^{\prime}+W_{z}^{\prime} & =0, \\
\Theta_{t}^{\prime}+D^{\prime} & =0,
\end{aligned}
$$

where

$$
D^{\prime}=\nabla \cdot \bar{\theta} \mathbf{V}^{\prime}=\bar{\theta}\left(U_{x}^{\prime}+W_{z}^{\prime}+\frac{N^{2}}{g} W^{\prime}\right)
$$

We then scale the prognostic variables using

$$
\left(U^{\prime}, W^{\prime}, \Theta^{\prime}, D^{\prime}\right)=\left(\frac{\bar{\rho}}{\rho_{0}}\right)^{1 / 2}(U, W, \bar{\theta} \Theta, \bar{\theta} D)
$$

to remove their dependence on vertical gradients in the mean atmosphere. The resulting linear equations for the scaled variables are

$$
\begin{aligned}
U_{t}+c^{2} \Theta_{x} & =\gamma_{h} D_{x}, \\
W_{t}+c^{2} \Theta_{z}-\frac{c^{2}}{2 H} \Theta+g \rho & =\gamma_{v}\left(D_{z}-\eta D\right), \\
\rho_{t}+U_{x}+W_{z}-\frac{1}{2 H} W & =0, \\
\Theta_{t}+D & =0,
\end{aligned}
$$

with

$$
D=U_{x}+W_{z}-\eta W \quad \text { and } \quad \eta=\frac{1}{2 H}-\frac{N^{2}}{g} .
$$

Here, $\gamma_{h}$ and $\gamma_{v}$ are the dimensional horizontal and vertical divergence damping coefficients, $H=R \bar{T} / g$ is the density scale height, and $N$ is the Brunt-Väisälä frequency. For an atmosphere with an isothermal mean state, all of the terms in (7)-(11) have constant coefficients.

To analyze the behavior of individual Fourier modes, the variables $\phi=(U, W, \rho, \Theta)$ are represented as

$$
\phi(x, z, t)=\hat{\phi} e^{i(k x+l z-\omega t)},
$$

where $(k, l)$ are the wavenumbers in the $(x, z)$ directions, and $\omega$ is the frequency. Substituting this
Fourier representation (12) into (7)-(10) yields a system of four linear homogeneous equations for which nontrivial solutions require that the $4 \times 4$ determinant of coefficients vanishes. Multiplying out this determinant equation produces a quartic equation for the frequency. Writing this equation in terms of dimensionless variables, the dispersion equation is thus given by

$\Omega^{4}+i\left(\delta^{2} \Lambda_{h}+\zeta^{2} \Lambda_{v}\right) \Omega^{3}-\Omega^{2}-i \Lambda_{h} \varepsilon^{2} \Omega+\varepsilon^{2}=0$,

where

$$
\begin{aligned}
& \Omega=\frac{\omega}{\omega_{S}}, \quad \omega_{S}=\kappa c, \quad \omega_{G}=\frac{k N}{\kappa}, \quad \varepsilon=\frac{\omega_{G}}{\omega_{S}}, \quad \delta=\frac{k}{\kappa}, \\
& \zeta=\frac{l+i \eta}{\kappa}, \quad \Lambda_{h}=\frac{\kappa \gamma_{h}}{c}, \quad \Lambda_{v}=\frac{\kappa \gamma_{v}}{c}, \quad \text { and } \\
& \kappa^{2}=k^{2}+l^{2}+\frac{1}{4 H^{2}} .
\end{aligned}
$$

We have also derived (13) from the equation sets using either $(\pi, \theta)$ or $(p, T)$ as prognostic variables. In assessing the acoustic and gravity wave frequencies inherent in (13), we follow GH07 in evaluating the coefficient of the $\Omega^{3}$ term, making the a posteriori approximation that $\kappa^{2} \simeq k^{2}+l^{2}$ and $i l \sim \partial / \partial z>1 / 2 H$, such that $\zeta^{2} \simeq 1-\delta^{2}$. Recognizing $\varepsilon$ as a small parameter, solutions to (13) for the acoustic mode frequency $\omega_{s}$ and the gravity wave frequency $\omega_{g}$ accurate to $O(\varepsilon)$ can be readily obtained:

$$
\begin{gathered}
\frac{\omega_{s}}{\omega_{S}}= \pm\left\{1-\frac{1}{4}\left[\delta^{2} \Lambda_{h}+\left(1-\delta^{2}\right) \Lambda_{v}\right]^{2}\right\}^{1 / 2} \\
-\frac{i}{2}\left[\delta^{2} \Lambda_{h}+\left(1-\delta^{2}\right) \Lambda_{v}\right]+O\left(\varepsilon^{2}\right), \\
\frac{\omega_{g}}{\omega_{G}}= \pm 1-\frac{i}{2}\left(1-\delta^{2}\right)\left(\Lambda_{h}-\Lambda_{v}\right) \varepsilon+O\left(\varepsilon^{2}\right) .
\end{gathered}
$$

At this order of approximation, (15) and (16) confirm that for $\gamma_{h}=\gamma_{v}, \omega_{g}$ is unaffected by the divergence damping, while the acoustic modes are damped by the imaginary part of the frequency $\omega_{s i}=-\kappa^{2} \gamma_{h} / 2$.

The frequency equation (13) is essentially the same as that derived by SK92 [their Eq. (34)] for a compressible Boussinesq atmosphere. GH07 derived a frequency equation for an acoustic mode filter based on divergence damping following the approach outlined by SK92, but for the linear compressible equations without making the compressible Boussinesq approximation. Applying the divergence damping using $D=\nabla \cdot \mathbf{v}$, they obtained the frequency equation [using the same 
notation as in (13) and (14) and the same approximation in the $\Omega^{3}$ term]:

$$
\begin{aligned}
& \Omega^{4}+i\left[\delta^{2} \Lambda_{h}+\left(1-\delta^{2}\right) \Lambda_{v}\right] \Omega^{3} \\
& -\Omega^{2}+\frac{g l}{N^{2}}\left(\Lambda_{h}-\Lambda_{v}\right) \varepsilon^{2} \Omega+\varepsilon^{2}=0 .
\end{aligned}
$$

Although GH07 indicated that the frequency equation derived by SK92 differed from theirs due to SK92's compressible Boussinesq approximation, in comparing (13) and (17), it is clear that the difference results from their damping of $D=\nabla \cdot \mathbf{v}$ for the compressible atmosphere instead of $D=\nabla \cdot \bar{\rho} \bar{\theta} \mathbf{v}$. Approximating solutions to (17) as a series expansion in the small parameter $\varepsilon$ yields

$$
\begin{aligned}
\frac{\omega_{g}}{\omega_{G}}= & \pm 1+\frac{1}{2}\left\{\frac{g l}{N^{2}}\left(\Lambda_{h}-\Lambda_{v}\right)+i\left[\delta^{2} \Lambda_{h}+\left(1-\delta^{2}\right) \Lambda_{v}\right]\right\} \varepsilon \\
& +O\left(\varepsilon^{2}\right),
\end{aligned}
$$

while the solution for $\omega_{s}$ is the same as (15). This expression for $\omega_{g}$ differs somewhat from $\mathrm{GH} 07$, as it includes the $O(\varepsilon)$ contribution from the $\Omega^{3}$ term in (17), and suggests that damping $D=\nabla \cdot \mathbf{v}$ may result in a weak instability of the gravity wave modes.

While applying divergence damping with $\gamma_{h}=\gamma_{v}$ provides robust damping of the acoustic modes, in splitexplicit or HEVI numerical integration schemes, it requires implicit treatment of the divergence damping term in the vertical momentum equation, which adds complexity to the numerical finite-difference equations. Implementing divergence damping on $D=\nabla \cdot \rho \theta \mathbf{v}$ in the horizontal only $\left(\gamma_{v}=0\right)$ has only $O(\varepsilon)$ effect on the gravity wave frequencies (16) and provides damping of the acoustic modes (15) given by $\omega_{s i}=-k^{2} \gamma_{h} / 2$, which is independent of the vertical wavenumber and is increasingly effective at higher wavenumbers on the horizontal mesh. As demonstrated by SK92, employing an acoustic filter as in (7) is equivalent to forming a horizontal diffusion equation for the divergence, with the amount of damping per time regulated by the dimensionless parameter $\alpha_{h}=\gamma_{h} \Delta t / \Delta x^{2}$.

\section{Acoustic filtering in the numerical finite- difference equations}

In constructing a divergence damping term in the horizontal momentum equation, it is beneficial to have a consistent numerical representation of the divergence, such that the divergence in the damping term in (7) has the same numerical form as the divergence in the $\Theta$ equation (10). In HEVI or split-explicit numerical schemes, the horizontal derivatives are typically evaluated using forward-backward differencing, such that the pressure gradient terms in the horizontal momentum equations are evaluated at the beginning of each time step, while the horizontal gradients in the equations for the thermodynamic variables are evaluated at the new time level, after the horizontal velocities have been advanced in time. The vertical derivatives are then averaged over the time step and computed using implicit numerics. The vertical derivative in the divergence $D$ in (10) thus contains implicit numerics, which complicates specifying a numerical representation of $D$ in the divergence-damping term in (7) that is consistent with the numerical form of $D$ in (10).

Implementation of an acoustic filter in the WRF Model and initially in MPAS is based on (7), but in a modified form to achieve consistency and avoid additional computation of the divergence term. Recognizing that the full divergence is essentially proportional to the negative time tendency of $\Theta$ as indicated in (10), (7) can be rewritten as

$$
U_{t}+c^{2}\left[\Theta^{t}+\alpha_{\Theta}\left(\Theta^{t}-\Theta^{t-\Delta t}\right)\right]_{x}=0
$$

(Klemp et al. 2007; Skamarock et al. 2008, 2012). In this form, it is apparent that the divergence damping is achieved by centering the horizontal pressure gradient slightly forward from time $t$ in the forward differencing for the horizontal gradient terms. In comparing (19) with the form of the divergence damping expressed in (7),

$$
\alpha_{\Theta}=\frac{\gamma_{h}}{c^{2} \Delta t}=\frac{\alpha_{h}}{\lambda_{x}^{2}}
$$

where $\lambda_{x}=c \Delta t / \Delta x$ is the horizontal acoustic Courant number. In the time-split numerics, the horizontal pressure gradient terms are evaluated on the small acoustic time steps, and thus the acoustic filter in (19) is not applied in the first small time step since $\Theta^{t-\Delta t}$ is not yet available for that set of small steps. With WRF and MPAS typically running 4-6 small time steps per large step, this technique has worked well in a wide variety of applications.

More recent adaptations of MPAS, however, have exposed deficiencies in employing divergence damping as expressed in (19). The current version of MPAS has the capability to run a time step for the dynamics that is smaller than that for the rest of the model system (scalar transport, physics, etc.). In this configuration, there might typically be two acoustic steps per dynamic time step and three dynamics steps per scalar/physics step. Using third-order Runge-Kutta for the dynamics time 
step, a total of four small time steps are then computed per dynamics time step, but only one of them (the second time step in the third Runge-Kutta stage) has $\Theta^{t-\Delta t}$ conveniently available for use in the filter, as represented in (19). The acoustic damping using (19) in MPAS also becomes more problematic in variable mesh applications. Since the time step is limited by the grid spacing in the fine-mesh region, the acoustic Courant number can be significantly smaller in the coarse-mesh region, which would require significantly greater forward centering of the horizontal pressure gradient in (19) [i.e. a larger value of $\alpha_{\Theta}$ in (20)] to achieve the same damping for a given $\alpha_{h}$.

To achieve consistency between the numerical form of the divergence in the acoustic filter and in the $\Theta$ equation, we include the divergence damping term as a final adjustment step in the numerical integration, such that the time integrations of the linear equations (7)-(11) are given by

$$
\begin{aligned}
U^{*+\Delta t} & =U^{t}-c^{2} \Delta t \Theta_{x}^{t}, \\
W^{t+\Delta t} & =W^{t}-c^{2} \Delta t\left(\bar{\Theta}_{z}^{t}-\frac{1}{2 H} \bar{\Theta}^{t}+\frac{g}{c^{2}} \bar{\rho}^{t}\right), \\
\rho^{t+\Delta t} & =\rho^{t}-\Delta t\left(U_{x}^{* t+\Delta t}+\bar{W}_{z}^{t}-\frac{1}{2 H} \bar{W}^{t}\right), \\
\Theta^{t+\Delta t} & =\Theta^{t}-\Delta t\left(U_{x}^{* t+\Delta t}+\bar{W}_{z}^{t}-\eta \bar{W}^{t}\right), \\
U^{t+\Delta t} & =U^{* t+\Delta t}+\gamma_{h} \Delta t\left(U_{x}^{* t+\Delta t}+\bar{W}_{z}^{t}-\eta \bar{W}^{t}\right)_{x} .
\end{aligned}
$$

Here, the time averaging is off-centered according to

$$
\bar{\phi}^{t}=\frac{1+\sigma}{2} \phi^{t+\Delta t}+\frac{1-\sigma}{2} \phi^{t},
$$

where $\phi$ represents any of the prognostic variables in (21)-(25).

Notice that combining (21) and (25) recovers the same equation as (7), and now the divergence term has the same numerical form as the divergence in (24). Thus, one can also think of this adjustment as being the first step in the next acoustic time step, applied in a manner that maintains a consistent finite difference form for the divergence. We will refer to this form of the divergence damping as the time-adjusted acoustic filter. Adding the divergence damping as an adjustment at the end of the time step also has the computational advantage that, rather than constructing the full divergence for use in (25), it can be readily recovered using $D=-\Theta_{t}$ from (24) after solving the vertically implicit equations (22)-(24).

To assess the damping characteristics of individual Fourier modes for this time-adjusted acoustic filter in the above equations (21)-(25), the prognostic variables $\phi=\left(U, U^{*}, \rho, W, \Theta\right)$ are represented as

$$
\phi(x, z)=A(k, l)^{m} \hat{\phi} e^{i(k x+l z)},
$$

where $A(k, l)$ is the amplification coefficient, and $m$ is the number of time steps. In this manner, $1-|A(k, l)|$ represents the fractional reduction in the amplitude of each Fourier mode per time step. Substituting (27) into (21)-(25) now produces a system of five linear homogeneous equations. Assuming C-grid staggering and representing spatial derivatives with secondorder centered finite differences, solving the $5 \times 5$ determinant of coefficients set equal to zero yields an amplitude equation expressed as a fourth-order polynomial for $A$ :

$$
\begin{aligned}
& {\left[\alpha_{h}(A-1)+\lambda_{x}^{2} A\right]\left[4(A-1)^{2}+\beta^{2}\left(\sigma^{+} A+\sigma^{-}\right)^{2}\right] S^{2}} \\
& +(A-1)^{4}+\left(\lambda_{z}^{2}+\xi \frac{\beta^{2}}{4}\right)(A-1)^{2}\left(\sigma^{+} A+\sigma^{-}\right)^{2}=0,
\end{aligned}
$$

where

$$
\begin{aligned}
\lambda_{x}=\frac{c \Delta t}{\Delta x}, \quad S=\sin \frac{k \Delta x}{2}, & \lambda_{z}=\frac{c \Delta t}{\Delta z} \sin \frac{l \Delta z}{2}, \\
\beta & =N \Delta t \cos \frac{l \Delta z}{2}, \quad \text { and } \quad \sigma^{ \pm}=1 \pm \sigma .
\end{aligned}
$$

Here, the coefficient $\xi$ represents the influence of the particular compressibility assumption. For the full linear compressible equations as represented by (21)-(25), $\xi=c_{p}^{2} /\left(4 R c_{v}\right) \simeq 1.2$ for an isothermal atmosphere. Deriving the corresponding amplitude equation for the compressible Boussinesq equations, as defined by Durran (2010) and as analyzed by SK92, yields the same equation as (28), except that $\xi=1$.

We consider first the behavior of pure acoustic modes without the presence of gravity waves $(\beta=0)$ and without off-centering of the vertically implicit time averaging $(\sigma=0)$. For this situation, (28) becomes a quadratic equation for $A$ representing the two acoustic modes. The amplification factors $|A|$ for the larger of the two acoustic roots are plotted in Fig. 1 as a function of the horizontal Courant number $\lambda_{x}$ for $\lambda_{z}=1,3$, and 10 . Here, we have also set $S=1$ (corresponding to the $2 \Delta x$ mode) because the highest horizontal wavenumbers exert the strongest constraint on the stability limits for a given $\lambda_{x}$. Notice that for a fixed $\lambda_{z}$, there is a range of $\lambda_{x}$ for which the $|A|$ is independent of $\lambda_{x}$ (and is the same for both roots). Within this range, the solution to (28) has the simple form

$$
|A|^{2}=1-4 \frac{\alpha_{h} S^{2}}{1+\lambda_{z}^{2}} .
$$



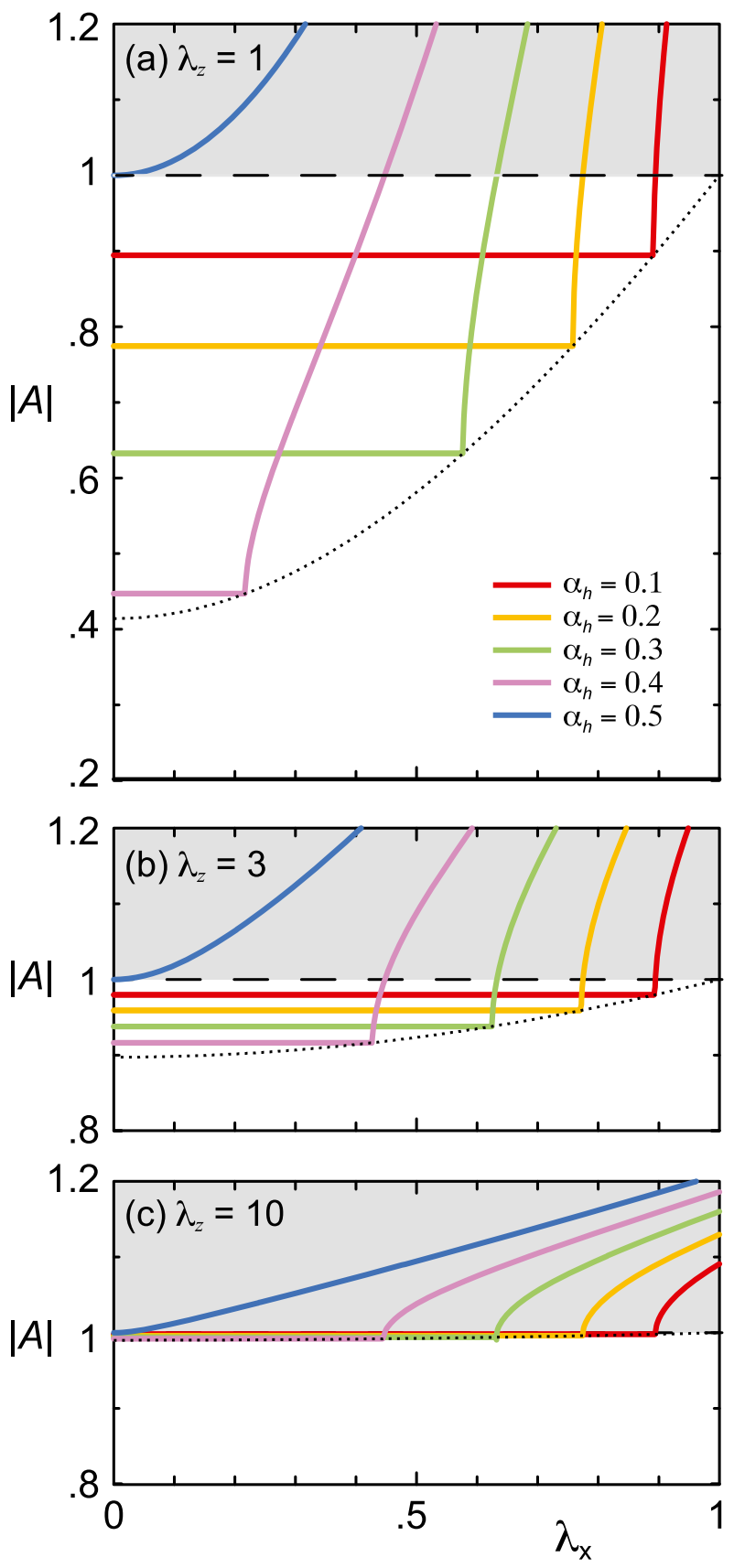

FIG. 1. Amplification factors $|A|$ for acoustic modes for $N=0$ for the time-adjusted acoustic filter in the HEVI scheme as represented in (21)-(25) as a function of $\lambda_{x}$ for $S=1$ and $\alpha_{h}=0.1-0.5$ for (a) $\lambda_{z}=1$, (b) $\lambda_{z}=3$, and (c) $\lambda_{z}=10$.

The thin dashed lines in Fig. 1 mark the boundary of the regions in which (30) applies and both roots have the same absolute value.

Further analysis of (28) reveals that for $\sigma=0$, this filter is stable (i.e., $|A|<1$ ) for $\alpha_{h}<\left(1-\lambda_{x}^{2}\right) / 2$, and this result is independent of the vertical Courant number $\lambda_{z}$.

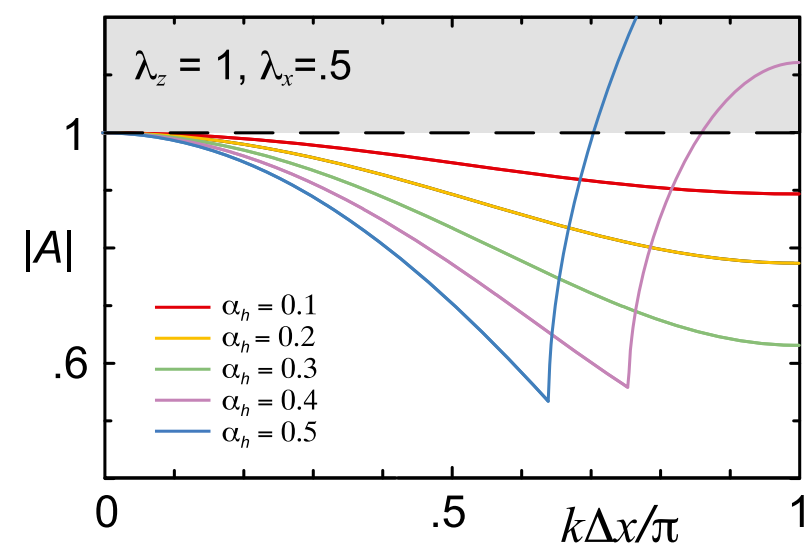

FIG. 2. Amplification factor $|A|$ for acoustic modes for $N=0$ for the time-adjusted acoustic filter in the HEVI scheme as represented in (21)-(25) as a function of horizontal wavenumber for $\lambda_{z}=1, \lambda_{x}=0.5$, and $\alpha_{h}=0.1-0.5$.

[For 3D domains, the stability limit for this divergence damping would be $\alpha_{h}<\left(1-\lambda_{x}^{2}-\lambda_{y}^{2}\right) / 2$.] Furthermore, for $\lambda_{z}>0$, the damping rate for a given horizontal mode $S$ is independent of the horizontal Courant number $\lambda_{x}$ over most of the range for which a given value of $\alpha_{h}$ is stable. As $\lambda_{z}$ increases, the damping provided by this filter decreases; for $\lambda_{z}$ as large as 10 (Fig. 1c), the filter provides essentially no damping of the acoustic modes. This behavior results from the vertically implicit numerics in the HEVI scheme and differs from that obtained for the analytic frequency equation, where the acoustic damping rate (15) was found to be independent of the vertical wavenumber. This is not problematic, however, since off-centering in the vertically implicit time step is expected to effectively filter acoustic modes for large $\lambda_{z}$.

As suggested by the amplification factor (30), for $\sigma=0$, the dependence of the damping rate on the horizontal scale for this divergence filter is approximately proportional to $S^{2}$, which is to be expected for a secondorder diffusion filter. This behavior is illustrated in Fig. 2 for $\lambda_{z}=1$ as in Fig. 1a, except now displaying $|A|$ as a function of the horizontal wavenumber for $\lambda_{x}=0.5$. For this value of $\lambda_{x},|A|>1$ at the higher wavenumbers for $\alpha_{h}=0.4$ and 0.5 , which is consistent with the abovementioned stability limit of $\alpha_{h}<\left(1-\lambda_{x}^{2}\right) / 2=0.375$.

The damping characteristics of the full acoustic/ gravity wave amplification equation (28) are illustrated in Fig. 3 for $\lambda_{x}=0.5, S=1$, and $\beta=0.25$, including offcentering in the vertically implicit numerics and the time-adjusted divergence damping term, applied both individually and together. In all three of these cases, damping of the gravity wave modes is very weak. With increasing $\beta$, the gravity wave damping increases 


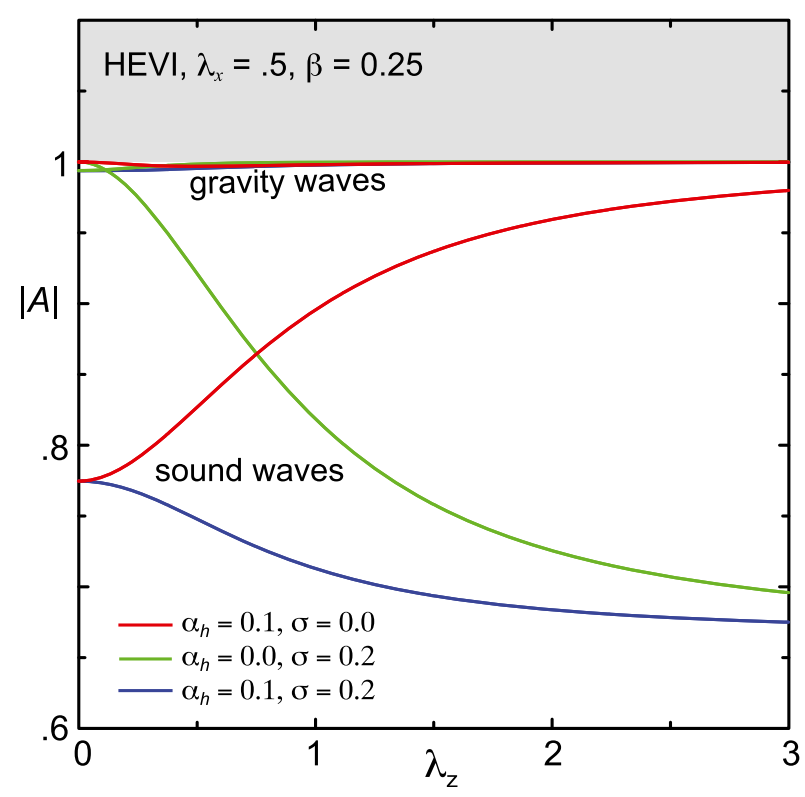

FIG. 3. Amplification factors $|A|$ for acoustic and gravity wave modes from (28) for $\lambda_{x}=0.5, S=1$, and $\beta=0.25$ for acoustic filtering with only off-centering in the vertically implicit numerics ( $\alpha_{h}=0, \sigma=0.2$ ), with only the time-adjusted divergence damping $\left(\alpha_{h}=0.1, \sigma=0\right)$, and with both vertical off-centering and divergence damping $\left(\alpha_{h}=0.1, \sigma=0.2\right)$.

somewhat at small values of $\lambda_{z}$. However, for HEVI and split-explicit numerics, increasing $\beta$ significantly implies larger values of $\Delta t$ (appropriate for coarser horizontal resolution), which also implies larger values of $\lambda_{z}$.

For the acoustic modes, the damping effects produced by the implicit vertical off-centering and the explicit horizontal divergence damping are quite different. With only the vertical off-centering, there is little acoustic damping as the vertical wavenumber approaches zero $\left(\lambda_{z} \rightarrow 0\right)$, but the damping increases rapidly with increasing $\lambda_{z}$. With only the horizontal divergence damping term, the acoustic damping behavior is reversed: the damping is a maximum at small $\lambda_{z}$ and decreases with increasing $\lambda_{z}$, as indicated by (30). With combined use of the vertical off-centering and the horizontal divergence damping, the damping effects are essentially additive and can provide significant acoustic damping for all $\lambda_{z}$. Since these acoustic modes are only weakly influenced by the atmospheric stability, the solution to (28) for $\beta=0$, but including the vertical off-centering,

$$
|A|^{2}=1-\frac{4\left(\alpha_{h} S^{2}+\sigma \lambda_{z}^{2}\right)}{1+(1+\sigma)^{2} \lambda_{z}^{2}}
$$

provides an accurate representation of the acoustic mode damping profiles shown in Fig. 3.

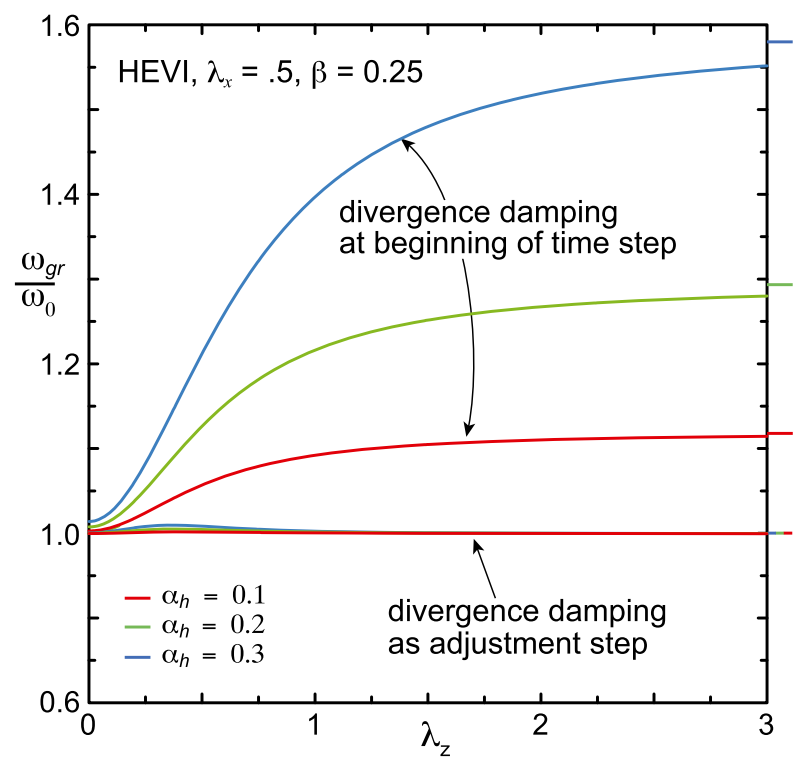

FIG. 4. Normalized gravity wave frequencies for the divergencedamping acoustic mode filter in a HEVI integration scheme with the damping term applied either at the beginning of the time step or as an adjustment at the end of the time step for $\lambda_{x}=0.5, S=1$, $\beta=0.25, \sigma=0$, and $\alpha_{h}=0.1,0.2,0.3$. Asymptotic values for large $\lambda_{z}$ from (34) are indicated to the right of the figure.

We note that vertically off-centering the implicit representation of the vertical pressure gradient in (22) can also be expressed in terms of the divergence as

$$
\begin{aligned}
\bar{\Theta}_{z}^{t} & =\left[\frac{1}{2}\left(\Theta^{t+\Delta t}+\Theta^{t}\right)+\frac{\sigma \Delta t}{2}\left(\frac{\Theta^{t+\Delta t}-\Theta^{t}}{\Delta t}\right)\right]_{z} \\
& \simeq \frac{1}{2}\left(\Theta^{t+\Delta t}+\Theta^{t}\right)_{z}-\frac{\sigma \Delta t}{2} D_{z} .
\end{aligned}
$$

Thus, this vertical off-centering is essentially equivalent to explicitly including a vertical divergence damping term in the vertical momentum equation, as in (3) or (8), with $\gamma_{v}=c^{2} \Delta t \sigma / 2$.

The real part of the gravity wave frequency $\omega_{g r}$ is physically important and therefore must not be significantly altered by an acoustic-mode filter. Applying the acoustic filter as an adjustment at the end of the time step as represented in (21)-(25) satisfies this requirement, as illustrated in Fig. 4, which displays the gravity wave frequency $\omega_{\mathrm{gr}}$ extracted from the gravity wave roots of (28) for $\lambda_{x}=0.5, S=1, \beta=0.25$, and $\sigma=0$ over a range of filter coefficients $\alpha_{h}=0.1,0.2$, and 0.3. Here, $\omega_{\mathrm{gr}}$ is computed using the relation $\omega_{\mathrm{gr}} \Delta t=\sin ^{-1}\left(A_{i} /|A|\right)$, where $A_{i}$ is the (positive) imaginary part of the gravity wave roots in (28) and is normalized in Fig. 4 by the gravity wave frequency $\omega_{0}$ with no filter $\left(\alpha_{h}=0\right)$. 
In applying the acoustic filter on every small time step, it is tempting to implement the filter as expressed in (7), with the divergence constructed using $D^{t}$ at the beginning of each acoustic time step. However, with this representation, the divergence would not be numerically the same as the finite difference form of the divergence term in the $\Theta$ equation (10) and therefore might not be effective in damping only the acoustic modes. To better understand the deficiencies of this approach, we also evaluated the dispersion equation for this filter [i.e., adding a term $\gamma_{h} D_{x}^{t}$ to the rhs of (21) and removing the adjustment step (25)]. In this case, the dispersion equation is as given by (28), except that it includes an additional term:

$$
-4 \alpha_{h} S^{2} \lambda_{z}^{2}(A-1)^{2} \sigma^{-}\left(\sigma^{+} A+\sigma^{-}\right) .
$$

The damping characteristics for this form of the filter appear to be similar to those for the time-adjusted filter behavior shown in Figs. 1-3; the linear stability constraints are essentially the same, and the gravity wave modes are weakly damped (not shown). However, there is a significant difference in the influence of the acoustic filter on the real part of the gravity wave frequency $\omega_{\mathrm{gr}}$. This is clearly evident in Fig. 4, which also displays $\omega_{\mathrm{gr}}$ when the acoustic filter is applied at the beginning of the time step (using $D^{t}$ ). With increasing $\alpha_{h}$, the gravity wave frequency $\omega_{\mathrm{gr}}$ departs increasingly from the correct value $\left(\omega_{0}\right)$, which corrupts the integrity of these physically important modes. For large values of $\lambda_{z}$, the asymptotic value of $\omega_{\mathrm{gr}}$ is readily obtained from (28) + (33). Recognizing that $\omega_{\mathrm{gr}}$ becomes $O\left(\lambda_{z}^{-1}\right)$ for large $\lambda_{z}$, letting $A=1-i \omega_{\mathrm{gr}} \Delta t$ in (28) + (33) and retaining only the $O(1)$ terms leads to

$$
\frac{\omega_{\mathrm{gr}}}{\omega_{0}}=\left(1-2 \alpha_{h} S^{2}\right)^{-1 / 2},
$$

which agrees closely with the limit of the values plotted in Fig. 4 as $\lambda_{z} \rightarrow \infty$. Given the desire to damp acoustic modes with minimal effect on the gravity waves, this form for the acoustic filter is not recommended. In practice, implementing the acoustic filter in this manner in the MPAS model produced inconsistent behavior in suppressing small-scale noise.

\section{Implementation of the acoustic filter in MPAS}

In MPAS, the dynamical equations are written in flux form in a height-based terrain-following vertical coordinate (Skamarock et al. 2012) and solve a prognostic equation for $\Theta_{m}=\rho_{d} \theta_{m}$ instead of a pressure equation, where $\rho_{d}$ is the density of dry air, and
$\theta_{m}=\left[1+\left(R_{v} / R_{d}\right) q_{v}\right] \theta$, such that $p=p_{0}\left(R_{d} \Theta_{m} / p_{0}\right)^{\gamma}$ with $\gamma=c_{p} / c_{v}$. The dynamical equations in MPAS are solved using a split-explicit time integration as described by Klemp et al. (2007), in which vertically propagating acoustic/gravity wave modes are treated using implicit numerics, and horizontally propagating modes are solved using explicit differencing, as indicated in the simplified linear equations (21)-(25) analyzed in the previous section. With this approach, the $\Theta_{m}$ equation that is advanced in time on the acoustic time steps becomes

$$
\Theta_{m}^{\tau+\Delta \tau}=\Theta_{m}^{\tau}-\Delta \tau\left\{\left[\nabla_{h} \cdot\left(\mathbf{V}_{h}^{* \tau+\Delta \tau} \theta_{m}^{t}\right)+\partial_{\zeta}\left(\bar{\Omega}^{\tau} \theta_{m}^{t}\right)\right]-F_{\Theta_{m}}^{t}\right\},
$$

where the notation corresponds to that used in Klemp et al. (2007). The divergence as represented by $D=\nabla \cdot \rho_{d} \theta_{m} \mathbf{v}$ is contained within the square brackets in (35) and evaluated on the acoustic time steps $(\Delta \tau)$, while $F_{\Theta_{m}}^{t}$ contains the physics forcing and other terms that are updated on the large time steps (denoted by $t$ ). For diabatic applications, Durran (1989) proposed a pseudoincompressible continuity equation $\nabla \cdot \bar{\rho} \bar{\theta} \mathbf{v}=H /\left(c_{p} \bar{\pi}\right)$ as an improvement to the traditional anelastic continuity equation, where $H$ is the diabatic heating per unit volume. This diabatic heating term is embedded within $F_{\Theta_{m}}^{t}$, and thus, applying the divergence damping filter to the terms within the large curly brackets in (35) may improve the numerical consistency in divergence damping for diabatic applications. Applying the acoustic filter as a final adjustment for the flux-form horizontal velocity $\mathbf{V}_{h}=\rho_{d} \mathbf{v}_{h}$ as in (25), this is easily accommodated without the need to calculate the full $3 \mathrm{D}$ divergence. Rather, the time derivative of $\Theta_{m}$ is used immediately after advancing (35), such that the divergence damping is added as a final adjustment step according to

$$
\mathbf{V}_{h}^{\tau+\Delta \tau}=\mathbf{V}_{h}^{*_{\tau}+\Delta \tau}-\frac{\alpha_{d}}{\theta_{m}^{t}} d^{2} \nabla_{h} \cdot\left(\frac{\Theta_{m}^{\tau+\Delta \tau}-\Theta_{m}^{\tau}}{\Delta \tau}\right),
$$

where on the MPAS mesh, $d$ represents the distance between cell centers adjacent to the edge on which the horizontal velocity is located and $\alpha_{d}=\gamma_{h} \Delta \tau / d^{2}$. In this form, the divergence is damped toward the diabatic heating rate (instead of zero), which is consistent with pseudoincompressible balance suggested by Durran (1989).

In applying a dimensionless acoustic filter coefficient $\alpha_{d}$ as in (36), a further adjustment is needed in configuring MPAS for variable-resolution meshes. For these applications, the spatially uniform time steps are specified to maintain stability in the regions of highest resolution. Consequently, the time steps in the coarserresolution regions will be significantly smaller than would be specified in a uniform mesh having that same 
coarse resolution. The uniform time step for a variableresolution mesh does not necessarily result in a large computational penalty since the majority of grid cells are often in the higher-resolution regions. However, it is desirable to scale the filter coefficients so that in the coarse-resolution regions of the mesh, they have the same influence on the flow as they would have in a uniform coarse-resolution run using a larger time step. To achieve this scaling, the dimensionless coefficient $\alpha_{d}=\gamma_{h} \Delta \tau / d_{f}^{2}$ is defined based on the mesh spacing in the fine-mesh region $d_{f}$ and is then locally adjusted by the factor $d_{f} / d$, where $d$ is the local cell size. This is readily accommodated in (36) by just replacing $d^{2}$ with $d d_{f}$.

\section{Acoustic filter in MPAS global forecasts}

In real-data applications of the global MPAS over a wide range of scales, acoustic filtering plays a crucial role not only in the numerical efficiency and stability, but also in the quality of the analysis and forecast. Fastmoving, small-scale acoustic modes can interact with uncertainties in the initial state to accelerate the error growth and ultimately limit the predictability of the simulated flow (Hohenegger and Schär 2007). Acoustic noise can be particularly detrimental in conjunction with ensemble data assimilation, as high-frequency noise in the background forecast can be significantly amplified by sampling error in the cycled ensemble analysis. Unless it is effectively suppressed during the model integration, such spurious noise remaining in the forecast can lead to a noisy analysis, which in turn can deteriorate the quality and the numerical stability of the ensuing forecast. The noise can continuously accumulate through cycles and speed up the forecast error growth at each cycle (and may eventually cause the forecast to crash). As Ha et al. (2017) demonstrated, without the effective noise filtering, even if the ensemble analysis was produced in a variable-resolution MPAS mesh, the forecast error grew quickly to counteract the benefit of the local refinement (e.g., forecasting in a highresolution mesh).

The surface pressure tendency reflects the vertically integrated mass divergence and is known to be especially sensitive to the presence of noise (McPherson et al. 1979). Previous studies of initialization schemes have evaluated the time evolution of surface pressure or the mean absolute tendency of surface pressure to measure the global noise levels (Williamson and Temperton 1981; Temperton and Williamson 1981; Lynch and Huang 1992; Huang and Lynch 1993). To test the behavior of the new acoustic filtering technique in MPAS as described in the previous section, global MPAS forecasts are initialized with the $1^{\circ} \times 1^{\circ}$ NCEP

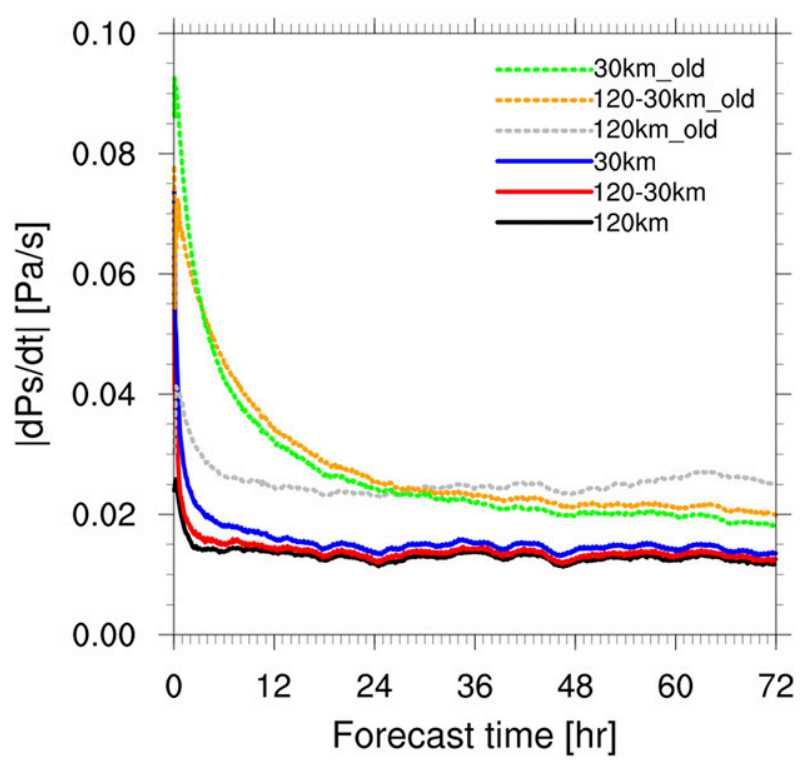

FIG. 5. Time series of the area-weighted global average of the absolute value of surface pressure tendency $\left(\mathrm{Pa} \mathrm{s}^{-1}\right)$. Each color represents an MPAS 3-day global forecast on different meshes using two different acoustic filtering methods, initialized with the same FNL analyses valid at 0000:00 UTC 15 Jun 2012. The 120-30-km variable-resolution mesh is configured with the local $30-\mathrm{km}$ refinement over the CONUS domain. The divergence damping is applied using the old method (dotted lines) and the new method (solid lines) as explained in the text.

Final (FNL) global analyses at 0000:00 UTC 15 June 2012 on three different meshes: $120-$ and $30-\mathrm{km}$ quasiuniform meshes and a $120-30-\mathrm{km}$ variable-resolution mesh in which a $120-\mathrm{km}$ global resolution is refined to a 30-km mesh spacing over the contiguous United States (CONUS). All the experiments have 55 vertical levels with the model top at $30 \mathrm{~km}$, and the model integration time steps are 720,180 , and $180 \mathrm{~s}$ for the simulations on 120-, 120-30-, and 30-km meshes, respectively. For comparison, simulations were also conducted with the previous version of the acoustic filter in MPAS, described at the beginning of section 3 .

To compare the high-frequency noise resulting from a cold-start model initialization, Fig. 5 shows the areaweighted global mean absolute tendency of surface pressure $\left(\mathrm{Pa} \mathrm{s}^{-1}\right)$ at every time step for 3-day forecasts using the two acoustic-filtering methods. In simulations labeled with "old" in Fig. 5, the divergence damping is applied by forward extrapolating the pressure gradient in the horizontal momentum equation as in (19), while the runs with the new acoustic filter applied as a final adjustment step as in (36) are simply shown by the mesh names. Both methods use the same filter coefficient $\alpha_{d}=\alpha_{\Theta}=0.1$, but the old approach does not contain the scaling factor for variable mesh spacing. In all 
experiments, the initial noise is large due to mass/wind imbalances in the initialization, but decreases through dynamical adjustment and damping during the model integration. The new filtering quickly suppresses noise over the first few time steps and then maintains a steady level after about $12 \mathrm{~h}$ into the forecast. These residual amplitudes should represent the tendencies produced by nonacoustic modes of physical interest. In contrast, with the old method, the initial noise decreases much more slowly (especially for the $30-\mathrm{km}$ uniform and $120-30-\mathrm{km}$ variable meshes) and asymptotes at higher levels. This suggests that there may be somewhat higher ongoing levels of acoustic noise in simulations with the old method. At the 72-h forecast, the asymptotes in the new filter are around $0.012 \mathrm{~Pa} \mathrm{~s}^{-1}$ for all three meshes, while the residual surface pressure tendency with the old technique is $50 \%-100 \%$ higher. Notice also that with the new filtering approach, the overall asymptotic amplitude of the surface pressure tendency is no longer sensitive to the mesh configuration, indicating that the filter is effective for variable-resolution meshes, regardless of the mesh resolution.

The new acoustic filter has been further tested on a much larger number of cases by running $120-\mathrm{km}$ mesh MPAS forecasts initialized at 0000 and 1200 UTC on the first day of each month every month for 5 years (120 cases). For these cases, the average amplitude of the surface pressure tendency for the 6-h forecasts is found to be about $0.015 \mathrm{~Pa} \mathrm{~s}^{-1}$ with little variance across cases. This asymptotic value for the MPAS forecasts is comparable to the ones presented in Lynch and Huang (1992) in their digital filter initialization (DFI) study. By applying a digital filter in the High-Resolution LimitedArea Model (HIRLAM) simulations at half-degree resolution, they obtained asymptotic values of mean absolute surface pressure tendency of $\sim 0.01 \mathrm{~Pa} \mathrm{~s}^{-1}$ in a 6-h forecast. Note that no initialization schemes such as DFI are applied in our MPAS runs to further reduce the noise caused by initial dynamical imbalances.

Figure 6 illustrates the horizontal distribution of the surface pressure tendency at three different forecast lead times for the 30-km uniform mesh using the old filtering technique. With the old acoustic filtering, spurious small-scale noise gradually decreases with time, but a significant amount still remains everywhere in the 6-h forecast. One interesting aspect of this case is the ring of large pressure tendencies emanating from the vicinity of the southern Philippine Sea. This disturbance is caused by the presence of Typhoon Guchol, which had category 2 intensity around the initialization time, but was poorly resolved in the $1^{\circ}$ NCEP FNL analysis. The acoustic character of these high-frequency waves is confirmed by the radial expansion of the disturbance ring,
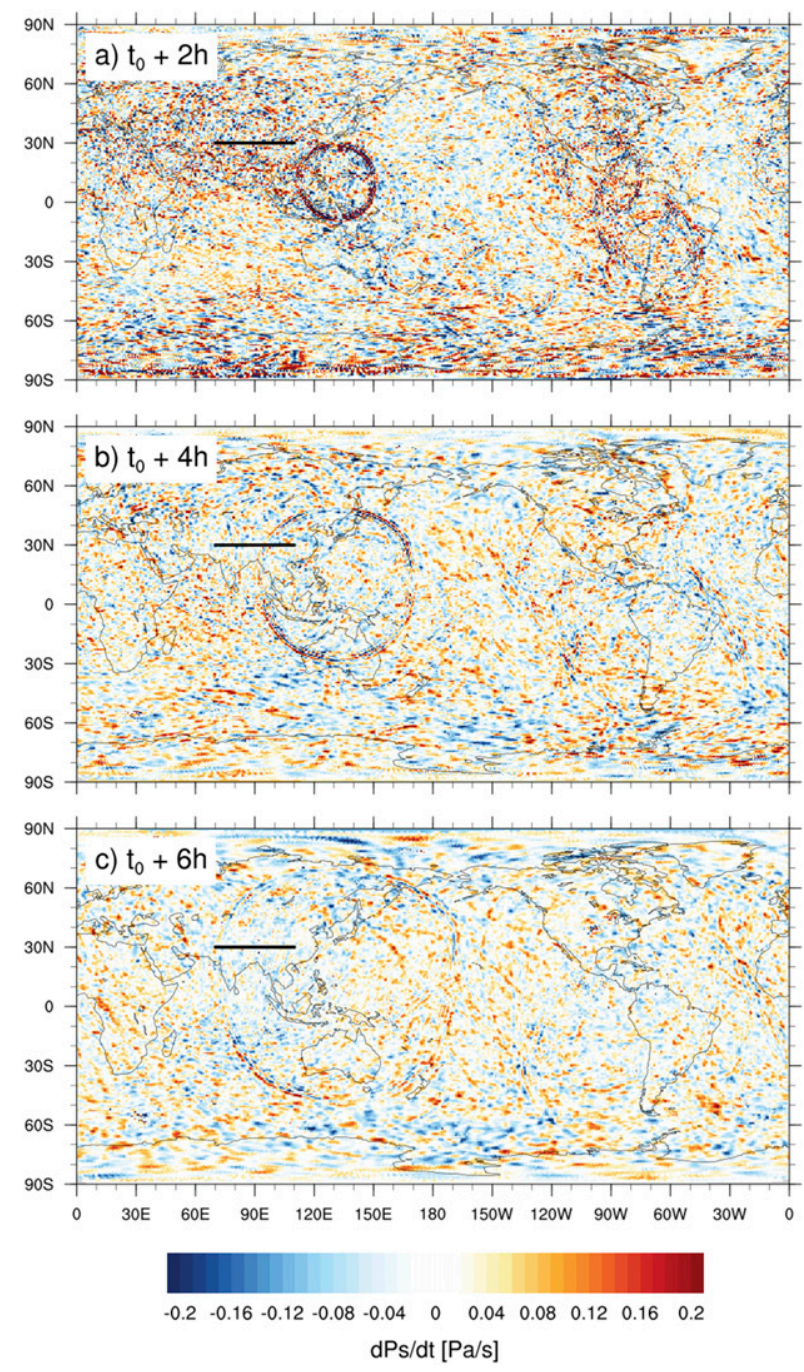

FIG. 6. Horizontal distribution of surface pressure tendency $\left(\mathrm{Pa} \mathrm{s}^{-1}\right)$ in the old filtering approach for the 30-km uniform mesh (e.g., "30 km_old") at (a) 2-, (b) 4-, and (c) 6-h forecast times, starting from the initialization time at 0000:00 UTC 15 Jun 2012.

with an estimated velocity greater than $305 \mathrm{~m} \mathrm{~s}^{-1}$. A second, weaker disturbance ring is also visible in Fig. 6a, centered off the southwest coast of Mexico. This acoustic noise is caused by Hurricane Carlotta, which was a category 2 storm around this time. These kinds of initial dynamic imbalances are a natural consequence of initializing a model forecast from a coarse-resolution analysis.

On the other hand, the new acoustic filtering method efficiently eliminates such high-frequency acoustic modes, as illustrated by the significantly reduced noise levels at $2 \mathrm{~h}$ shown in Fig. 7. (Comparable plots at 4 and $6 \mathrm{~h}$ are not shown, as the noise levels at $2 \mathrm{~h}$ are already so much lower than those obtained with the old filtering approach at those later times.) Because mesoscale analysis/forecast cycling typically runs at the frequency 


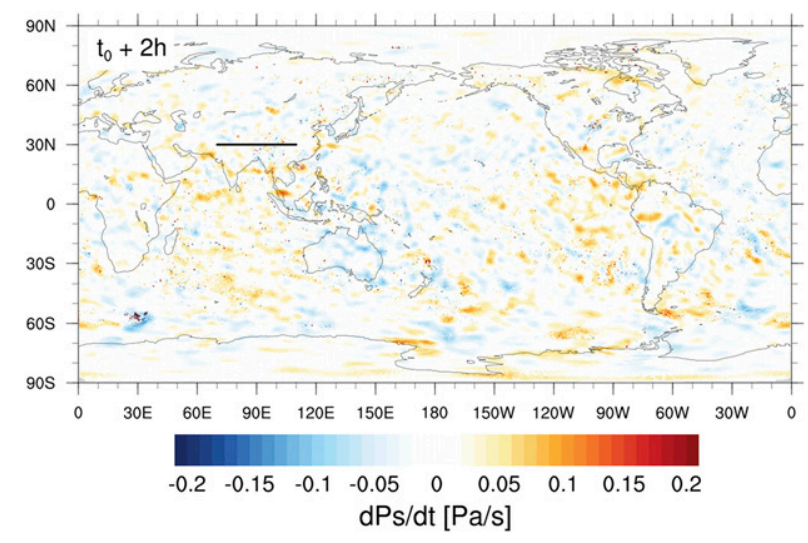

FIG. 7. As in Fig. 6a, but for the new acoustic filtering method with the $30-\mathrm{km}$ mesh at $2 \mathrm{~h}$.

of $6 \mathrm{~h}$ (or shorter), the noise remaining in the 6-h forecast can be closely associated with the forecast reliability and skill throughout the cycles. Therefore, the efficient suppression of high-frequency oscillations is particularly important at such short forecast lead times in mesoscale applications.

Figure 8 depicts vertical velocity $\left(\mathrm{m} \mathrm{s}^{-1}\right)$ at the 6 -h forecast time in a vertical cross section over the Himalayas along the $30^{\circ} \mathrm{N}$ latitude line, as marked in Figs. 6 and 7. In comparing the two filtering methods, it is evident that vertical velocity perturbations are significantly smaller with the new approach (Fig. 8b), with the maximum amplitudes reduced by a factor of 2-3. Evaluating the time evolution of these perturbations (not shown), two main features are found: (i) the stronger upward motions (in orange) are triggered over the high topography around $5 \mathrm{~h}$ into the forecast, about the time the acoustic waves originating from the tropical storm traverse this high terrain area, and (ii) the waves appear to be quasi stationary, oscillating slowly over the next $6 \mathrm{~h}$ with maximum amplitudes in the range of $1-3 \mathrm{~m} \mathrm{~s}^{-1}$. Thus, it appears that acoustic noise caused by dynamical imbalances in the model initialization is artificially amplifying atmospheric waves that would be of physical interest in this real-data forecast. With the new acoustic filter (Fig. 8b), the excitation of perturbations over the Himalayas is much weaker, with the maximum vertical velocities remaining below $0.5 \mathrm{~m} \mathrm{~s}^{-1}$ over the first $12 \mathrm{~h}$.

It should be also mentioned that when the acoustic filter is turned off altogether, the rapid growth of instabilities from the initial imbalances causes the model simulation to blow up in just under a 5-h integration time. Uncontrolled small-amplitude acoustic waves imposed on the initial conditions are quickly excited while propagating throughout the entire atmosphere and nonlinearly interacting with gravity (and inertia-gravity)
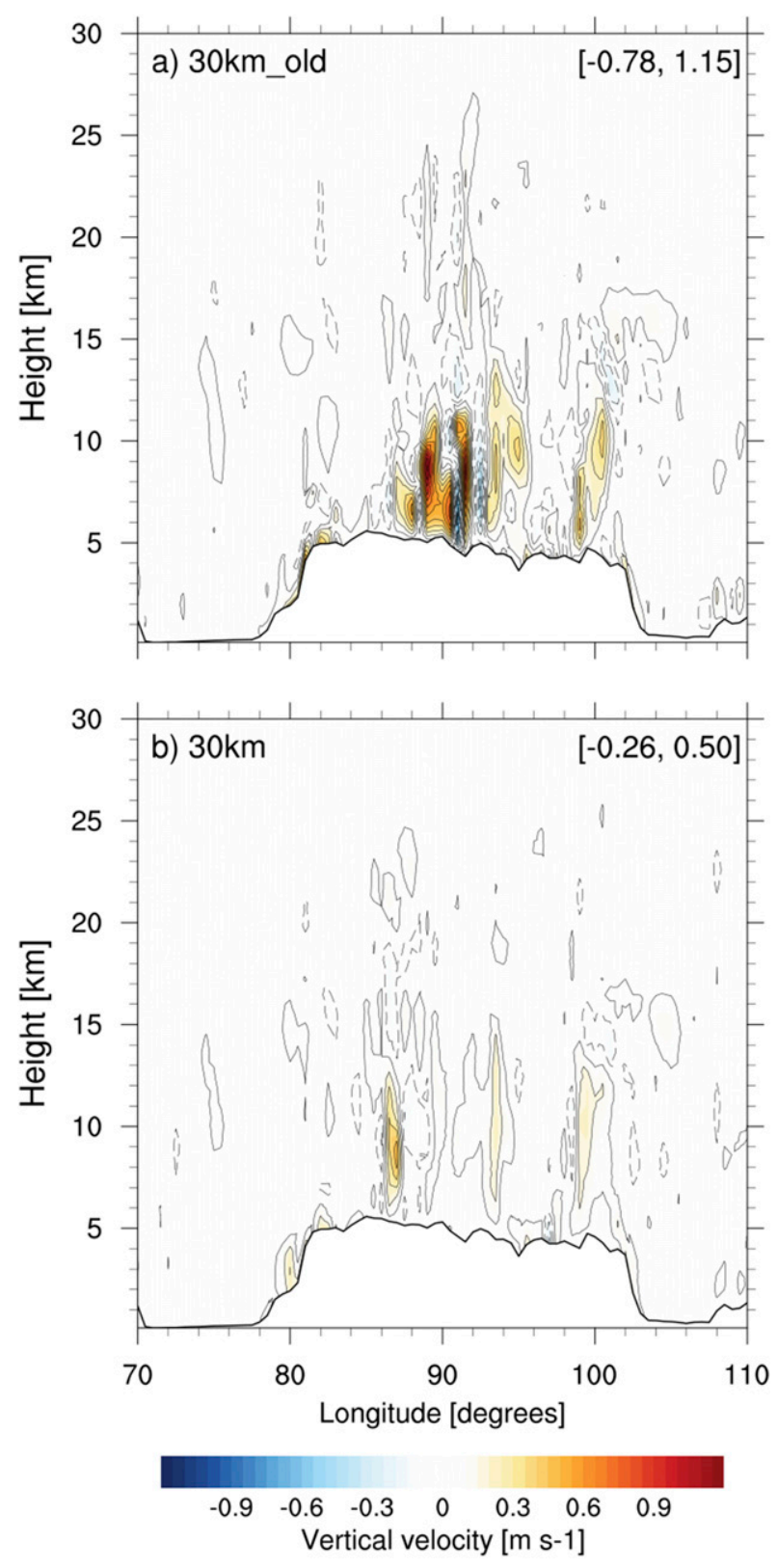

FIG. 8. Cross sections of vertical velocity $\left(\mathrm{m} \mathrm{s}^{-1}\right)$ at $6 \mathrm{~h}$ along the $30^{\circ} \mathrm{N}$ line marked in Figs. 6 and 7 in the 30-km uniform mesh simulations with (a) old and (b) new acoustic filtering. Minimum and maximum values are shown within brackets in the upper-right corner. Contours range from -1.15 to $1.15 \mathrm{~m} \mathrm{~s}^{-1}$ at $0.1 \mathrm{~m} \mathrm{~s}^{-1}$ interval. Downward motions are contoured in dashed lines.

waves to reach amplitudes that exceed the numerical stability limit.

\section{Summary}

In filtering acoustic modes by damping the full 3D divergence, the characteristics of the filter will depend 
on how the divergence is defined. For the adiabatic compressible atmosphere, based on the form of the pressure equation as expressed in (1), we have found that the expression for divergence that is most specific to the acoustic modes is given by $D=\nabla \cdot \rho \theta \mathbf{v}$. This form is also consistent with the pseudoincompressible approximation $\nabla \cdot \bar{\rho} \bar{\theta} \mathbf{v}=H /\left(c_{p} \bar{\pi}\right)$ proposed by Durran (1989) as an improvement to the traditional anelastic approximation. As we have demonstrated, defining the 3D divergence in this manner, applying divergence damping only in the horizontal avoids adverse effects on gravity waves.

In numerically computing the horizontal divergence damping terms, we find that it is important that the numerical representation of the divergence used in the damping terms is numerically the same as the numerical form of the divergence contained in the $\Theta=\rho \theta$ (or pressure) equation. We achieve this consistency by employing divergence damping as a final adjustment to the horizontal momentum equations after the divergence has been evaluated in the $\Theta$ equation. Using the time tendency of $\Theta$ to represent the divergence in this adjustment step, as indicated in (36), avoids redundant calculation of the $3 \mathrm{D}$ divergence. It also includes the diabatic effects $F_{\Theta_{m}}^{t}$ contained in the $\Theta$ equation (35), which is consistent with Durran's (1989) pseudoincompressible approximation that includes diabatic heating.

As illustrated by the real-data MPAS simulations in section 5, the model forecasts at early times may be severely contaminated by high-frequency oscillations as a result of dynamic imbalances in the initial conditions, which are typically interpolated from coarse-resolution analyses in cold-start simulations. With the acoustic filter applied as a final adjustment in the acoustic time step as indicated in (36), this high-frequency noise is rapidly attenuated and asymptotes to low residual values that do not appear to be sensitive to the mesh configuration. With the old acoustic filter, applied as in WRF based on (19), this acoustic noise is damped out much more slowly and asymptotes to higher values that are more sensitive to the specific mesh configuration.

It is not unexpected that the horizontal divergence damping technique used in the WRF Model is not sufficient to control acoustic noise in MPAS. As mentioned above, in the split-explicit numerics, the horizontal filter (19) is only applied after the first acoustic time step, which limits its application in the current configuration of MPAS that employs only two acoustic steps per dynamic time step. In addition, the WRF Model is less dependent on a 3D divergence filter since it also contains an external mode filter, as described by Skamarock et al. (2008), that effectively damps external modes that have large vertical wavelength. These external modes can be excited in the WRF Model as a result of its pressurebased sigma coordinate used in conjunction with a constant pressure boundary condition along the top of the model domain. The global MPAS uses a terrain-following height coordinate with a rigid-lid upper boundary, which does not support external modes, so this additional filter is not present in the MPAS numerics.

Acknowledgments. The National Center for Atmospheric Research is sponsored by the National Science Foundation, and funding for this research was provided through support from the National Science Foundation under Cooperative Support Agreement AGS-0856145.

\section{REFERENCES}

Doms, G., and M. Baldauf, 2015: A description of the Nonhydrostatic Regional COSMO-Model. Part I: Dynamics and numerics. Consortium for Small-Scale Modelling Rep., 155 pp., http://www.cosmo-model.org/content/model/documentation/ core/cosmoDyncsNumcs.pdf.

Dudhia, J., 1993: A nonhydrostatic version of the Penn StateNCAR Mesoscale Model: Validation tests and simulation of an Atlantic cyclone and cold front. Mon. Wea. Rev., 121, 1493-1513, https://doi.org/10.1175/1520-0493(1993)121<1493: ANVOTP $>2.0 . \mathrm{CO} ; 2$.

Durran, D. R., 1989: Improving the anelastic approximation. J. Atmos. Sci., 46, 1453-1461, https://doi.org/10.1175/1520-0469(1989)046<1453: ITAA $>2.0 . \mathrm{CO} ; 2$.

_ 2010: Numerical Methods for Fluid Dynamics. SpringerVerlag, $516 \mathrm{pp}$.

__ , and J. B. Klemp, 1983: A compressible model for the simulation of moist mountain waves. Mon. Wea. Rev., 111, 2341-2361, https://doi.org/10.1175/1520-0493(1983)111<2341: ACMFTS $>2.0 . \mathrm{CO} ; 2$.

Gassmann, A., 2005: An improved two-time-level split-explicit integration scheme for non-hydrostatic compressible models. Meteor. Atmos. Phys., 88, 23-38, https://doi.org/10.1007/ s00703-003-0053-8.

— , and H.-J. Herzog, 2007: A consistent time-split numerical scheme applied to the nonhydrostatic compressible equations. Mon. Wea. Rev., 135, 20-36, https://doi.org/10.1175/ MWR3275.1.

Ha, S., C. Snyder, W. C. Skamarock, J. L. Anderson, and N. Collins, 2017: Ensemble Kalman filter data assimilation for the Model for Prediction Across Scales (MPAS). Mon. Wea. Rev., 145, 4673-4692, https://doi.org/10.1175/MWR-D-17-0145.1.

Hodur, R. M., 1997: The Naval Research Laboratory's Coupled Ocean/Atmosphere Mesoscale Prediction System (COAMPS). Mon. Wea. Rev., 125, 1414-1430, https://doi.org/10.1175/ 1520-0493(1997)125<1414:TNRLSC >2.0.CO;2.

Hohenegger, C., and C. Schär, 2007: Predictability and error growth dynamics in cloud- resolving models. J. Atmos. Sci., 64, 4467-4478, https://doi.org/10.1175/2007JAS2143.1.

Huang, X.-Y., and P. Lynch, 1993: Diabatic digital-filtering initialization: Application to the HIRLAM Model. Mon. Wea. Rev., 121, 589-603, https://doi.org/10.1175/1520-0493(1993)121<0589: DDFIAT $>2.0 . \mathrm{CO} ; 2$.

Klemp, J. B., and R. B. Wilhelmson, 1978: The simulation of threedimensional convective storm dynamics. J. Atmos. Sci., 35, 
1070-1096, https://doi.org/10.1175/1520-0469(1978)035<1070: TSOTDC $>2.0 . \mathrm{CO} ; 2$.

- W. C. Skamarock, and J. Dudhia, 2007: Conservative splitexplicit time integration methods for the compressible nonhydrostatic equations. Mon. Wea. Rev., 135, 2897-2913, https://doi.org/10.1175/MWR3440.1.

Kurowski, M. J., W. W. Grabowski, and P. K. Smolarkiewicz, 2014: Anelastic and compressible simulation of moist deep convection. J. Atmos. Sci., 71, 3767-3787, https://doi.org/10.1175/ JAS-D-14-0017.1.

Lipps, F. B., and R. S. Hemler, 1982: A scale analysis of deep moist convection and some related numerical calculations. J. Atmos. Sci., 39, 2192-2210, https://doi.org/10.1175/1520-0469(1982) $039<2192$ :ASAODM $>2.0 . \mathrm{CO} ; 2$.

Lock, S.-J., N. Wood, and H. Weller, 2014: Numerical analyses of Runge-Kutta implicit-explicit schemes for horizontally explicit, vertically implicit solutions of atmospheric models. Quart. J. Roy. Meteor. Soc., 140, 1654-1669, https:// doi.org/10.1002/qj.2246.

Lynch, P., and X.-Y. Huang, 1992: Initialization of the HIRLAM Model using a digital filter. Mon. Wea. Rev., 120, 1019-1034, https://doi.org/10.1175/1520-0493(1992)120<1019: IOTHMU $>2.0 . \mathrm{CO} ; 2$.

McPherson, R. D., K. H. Bergman, R. E. Kistler, G. E. Rasch, and D. S. Gordon, 1979: The NMC Operational Global Data Assimilation System. Mon. Wea. Rev., 107, 1445-1461, https:// doi.org/10.1175/1520-0493(1979)107<1445:TNOGDA>2.0.CO;2.

Ogura, Y., and N. A. Phillips, 1962: Scale analysis of deep and shallow convection in the atmosphere. J. Atmos. Sci., 19, 173-179, https://doi.org/10.1175/1520-0469(1962)019<0173: SAODAS $>2.0 . \mathrm{CO} ; 2$.

Satoh, M., T. Matsuno, H. Tomita, H. Miura, T. Nasuno, and S. Iga, 2008: Nonhydrostatic icosahedral atmospheric model (NICAM) for global cloud resolving simulations. J. Comput. Phys., 227, 3486-3514, https://doi.org/10.1016/j.jcp.2007. 02.006 .

Skamarock, W. C., and J. B. Klemp, 1992: The stability of time-split numerical methods for the hydrostatic and the nonhydrostatic elastic equations. Mon. Wea. Rev., 120, 2109-2127, https:// doi.org/10.1175/1520-0493(1992) $120<2109$ :TSOTSN $>2.0$. $\mathrm{CO} ; 2$. and Coauthors, 2008: A description of the Advanced Research WRF version 3. NCAR Tech. Note NCAR/TN-475+STR, 113 pp., https://doi.org/10.5065/D68S4MVH.

_ J. B. Klemp, M. G. Duda, L. D. Fowler, S.-H. Park, and T. D. Ringler, 2012: A multiscale nonhydrostatic atmospheric model using centroidal Voronoi tessellations and C-grid staggering. Mon. Wea. Rev., 140, 3090-3105, https://doi.org/10.1175/ MWR-D-11-00215.1.

Staniforth, A., and J. Côté, 1991: Semi-Lagrangian integration schemes for atmospheric models-A review. Mon. Wea. Rev., 119, 2206-2223, https://doi.org/10.1175/1520-0493(1991)119<2206: SLISFA $>2.0 . \mathrm{CO} ; 2$.

Tanguay, M., A. Robert, and R. Laprise, 1990: A semi-implicit semi-Lagrangian fully compressible regional forecast model. Mon. Wea. Rev., 118, 1970-1980, https://doi.org/10.1175/1520-0493(1990) $118<1970$ :ASISLF $>2.0$. CO;2.

Tapp, M. C., and P. W. White, 1976: A non-hydrostatic mesoscale model. Quart. J. Roy. Meteor. Soc., 102, 277-296, https:// doi.org/10.1002/qj.49710243202.

Temperton, C., and D. Williamson, 1981: Normal mode initialization for a multilevel grid-point model. Part I: Linear aspects. Mon. Wea. Rev., 109, 729-743, https://doi.org/10.1175/1520-0493(1981) $109<0729$ :NMIFAM $>2.0 . C O ; 2$.

Wilhelmson, R. B., and Y. Ogura, 1972: The pressure perturbation and the numerical modeling of a cloud. J. Atmos. Sci., 29, 1295-1307, https://doi.org/10.1175/1520-0469(1972)029<1295: TPPATN $>2.0 . \mathrm{CO} ; 2$.

Williamson, D., and C. Temperton, 1981: Normal mode initialization for a multilevel grid-point model. Part II: Nonlinear aspects. Mon. Wea. Rev., 109, 744-757, https://doi.org/10.1175/ 1520-0493(1981)109<0744:NMIFAM > 2.0.CO;2.

Xue, M., K. K. Droegemeier, and V. Wong, 2000: The Advanced Regional Prediction System (ARPS)-A multi-scale nonhydrostatic atmospheric simulation and prediction model. Part I: Model dynamics and verification. Meteor. Atmos. Phys., 75, 161-193, https://doi.org/10.1007/s007030070003.

Zängl, G., D. Reinert, P. Rípodas, and M. Baldauf, 2015: The ICON (Icosahedral Non-hydrostatic) modelling framework of DWD and MPI-M: Description of the non-hydrostatic dynamical core. Quart. J. Roy. Meteor. Soc., 141, 563-579, https:// doi.org/10.1002/qj.2378. 